\title{
Opportunistic Scheduling and Adaptive Modulation in Wireless Networks with Network Coding
}

\author{
Seong-Lyong Gong \\ School of Electrical and \\ Electronic Engineering \\ Yonsei University, Seoul, Korea \\ Email: slgong@yonsei.ac.kr
}

\author{
Byung-Gook Kim \\ School of Electrical and \\ Electronic Engineering \\ Yonsei University, Seoul, Korea \\ Email: maum0312@yonsei.ac.kr
}

\author{
Jang-Won Lee \\ School of Electrical and \\ Electronic Engineering \\ Yonsei University, Seoul, Korea \\ Email: jangwon@yonsei.ac.kr
}

\begin{abstract}
So far, many researches on network coding are performed with higher layer protocols such as MAC, routing, and flow control protocols without consideration of physical layer issues such as channel conditions of links. However, in wireless networks, the consideration of properties at the physical layer is important to improve system performance. Hence, in this paper, we study an opportunistic scheduling and adaptive modulation problem for wireless networks with network coding, which is a joint problem for MAC and physical layers. A similar problem was studied in [1] considering an idealized system in which the data rate of each link is modeled with the Shannon capacity. They showed that to maximize the throughput of a transmission, the optimal subset of native packets that are encoded within a coded packet should be selected based on the channel condition at the destination for each native packet. Moreover, they also showed that it may not be the optimal selection to encode all possible native packets within a coded packet. In this paper, we consider a more realistic model than that of [1] with practical modulation schemes such as M-PSK and MQAM. We show that the optimal policy that maximizes the throughput of a transmission is to encode all available native packets within a coded packet regardless of the channel condition at the destination for each native packet, which is a different conclusion from that of [1]. However, we show that adaptive modulation, in which its constellation size in a coded packet is adjusted based on the channel condition of each destination node, provides a higher throughput than the scheme with fixed modulation, in which its constellation size is always fixed regardless of the channel condition at each destination node.
\end{abstract}

Index Terms-Wireless networks, Network coding, Scheduling, Adaptive modulation.

\section{INTRODUCTION}

Since the publication by Ahlswede et. al. [2], network coding has emerged as a promising solution to achieve higher throughput in both wireline and wireless networks. In the traditional network without network coding, a node forwards packets that it received to the appropriate next hop node without any processing in the information part of the packet. However, in the network with network coding, a node forwards coded packets produced by performing some processing with several packets that it has already received. Even though network coding requires coding and decoding processes additionally in each node in the network, it has been shown

This work was supported by the Korea Science and Engineering Foundation(KOSEF) grant funded by the Korea government(MEST) (No. R01-2008000-20578-0)

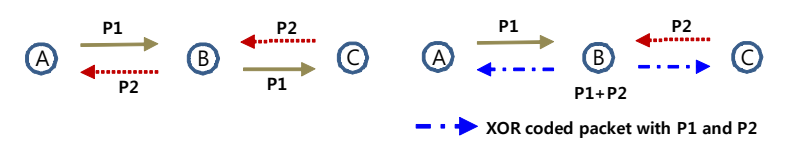

(a) Packet exchanges without (b) Packet exchanges with XOR XOR coding. coding.

Fig. 1. An example of for the XOR-based network coding scheme.

that if we use the appropriate network coding scheme, we can improve network performance significantly [2], [3], [4], [5]. Especially, in wireless network, if we appropriately exploit the broadcasting nature of wireless transmissions with network coding, we can improve network throughput more significantly by reducing the number of required transmissions [3], [4].

In Fig. 1, there are three nodes that can transmit packets only to adjacent nodes. Nodes $A$ and $C$ want to exchange their packets $P 1$ and $P 2$ to each other through node $B$. In this case, we can easily see that four transmissions are required in the traditional network without network coding, as in Fig. 1(a). We now show how network coding can reduce the number of transmissions in the wireless network with a simple XOR network coding scheme, in which coding and decoding processes are done with simple bitwise XOR operations with several packets. As in Fig. 1(b), nodes $A$ and $C$ first transmit their packets $P 1$ and $P 2$ to node $B$. Even after transmitting their packets, nodes $A$ and $C$ keep their own packets for a while without deleting them. After receiving packets $P 1$ and $P 2$, node $B$ makes a coded packet, $P 1+P 2$, by performing bitwise XOR with packets $P 1$ and $P 2$, and then node $B$ broadcasts coded packet $P 1+P 2$ to nodes $A$ and $C$. After receiving coded packet $P 1+P 2$, node $A$ can retrieve native packet $P 2$ destined to itself by performing bitwise XOR with coded packet $P 1+P 2$ and its own packet $P 1$. In a similar way, node $C$ can retrieve native packet $P 1$ destined to itself. Hence, with XOR network coding, total three transmissions are required, reducing one transmission compared with the case without network coding.

Even though this XOR network coding is simple, if we use it appropriately, we can improve the throughput of the wireless network. In [5], [6], COPE, which is a protocol between the IP and MAC layer with XOR network coding 


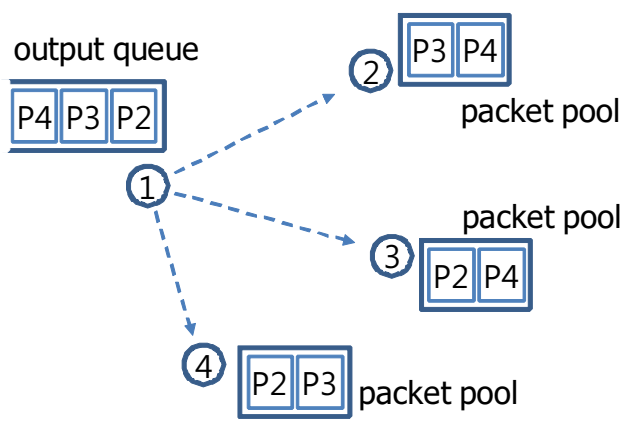

Fig. 2. Opportunistic coding in COPE.

for wireless ad-hoc networks, is proposed. In this protocol, each node stores packets that it overheard even though they are not destined to it and periodically reports to its neighbor nodes which packets it currently stores. Hence, each node has information on packets that each of its neighbor nodes stores. By using this information, a node can encode several native packets together, which results in further reducing the number of required transmissions.

For example, node 1 in Fig. 2 has three native packets, from $P 2$ to $P 4$ in its output queue. It wants to send packet $P 2$ to node 2 , packet $P 3$ to node 3 , and packet $P 4$ to node 4 . Node 2 has packets $P 3$ and $P 4$, node 3 has packets $P 2$ and $P 4$, and node 4 has packets $P 2$ and $P 3$. Node 1 knows this information through reports from nodes 2,3 , and 4 . In this case, there exist several coding options and it is important to select the optimal coding option. If node 1 performs XOR network coding with native packets $P 2$ and $P 3$ and broadcasts coded packet $P 2+$ $P 3$, nodes 2 and 3 can decode the coded packet and retrieve native packets that are destined to themselves. Hence, node 1 can send two native packets with one transmission. On the other hand, if node 1 performs XOR network coding with native packets $P 2, P 3$, and $P 4$ and broadcasts coded packet $P 2+P 3+P 4$, then nodes 2,3 , and 4 can decode the coded packet to retrieve native packets destined to them. Hence, in this case, node 1 can send three packets with one transmission and this is the optimal coding option. This choosing procedure for the optimal option is called opportunistic coding in COPE and it has been shown that opportunistic coding can improve network throughput significantly.

In COPE, we can easily see that the optimal coding is to include as many native packets as possible that can be decoded at each of their destination nodes within a coded packet. Referring to [1] in which the set of destination nodes in this option is defined as the network coding group, we call this coding option the MAC-optimal coding option. Hence, the network coding group consists of the largest possible number of destination nodes that satisfy below three conditions.

1: All the $N$ destination nodes in the network coding group are in transmission range of the transmitter node.

2: The transmitter node has $N$ native packets each of which is destined to each of destination nodes.

3: Each destination node knows other $N-1$ native packets excepting the native packet destined to itself.

Hence, if there is no error in the coded packet at each destination node, each destination node can retrieve the native packet destined to itself by performing bitwise XOR with the coded packet and $N-1$ native packets that it has.

In wireless network protocols, it is well known that the consideration of time-varying and location-dependent channel condition is very important to improve network performance. Even though [5], [6] proposed a nice protocol with simple XOR network coding that improves network throughput, it considered only the MAC layer without considering timevarying and location-dependent channel conditions of links at the physical layer together. Recently, this issue was studied by [1] through opportunistic scheduling, in which a node determines native packets that are encoded together, i.e., the destination nodes of the coded packet, with considering the channel condition at each destination node. In [1] the Shannon capacity is used for the capacity of each link and it is assumed that data is transmitted without any error if the data rate of a link is less than or equal to its capacity and otherwise, data cannot be transmitted at all. This assumption in [1] is somewhat unrealistic, since in practice, in any cases, there are a certain positive probability that errors occur in transmission and also a certain positive probability that errors do not occur. With this idealized model, [1] showed that opportunistic scheduling, in which only native packets of a subset of destination nodes in the network coding group are dynamically selected for a coded packet considering the channel condition at each destination node is the optimal policy.

In this paper, we study opportunistic scheduling and adaptive modulation in the network coding group, which is a similar problem to that of [1]. However, in this paper, we study this problem with a more realistic model than [1], considering practical modulation schemes such as M-PSK and MQAM and their error probabilities. With this model, in fact, we make a conclusion that is different from the conclusion of [1]. To maximize throughput of a transmission, we should include all native packets for all destination nodes in the network coding group. In order words, the MAC-optimal coding option is still optimal even though we consider the channel conditions at destination nodes. However, we also show that adaptive modulation, in which its constellation size is dynamically adapted to the the channel condition at each destination node in the network coding group, provides higher throughput than fixed modulation, in which its constellation size is always fixed regardless of the channel condition at each destination node. Hence, to maximize the throughput of a transmission, we only have to have the adaptive modulation that considers channel conditions at destination nodes without packet scheduling for network coding.

The rest of this paper is organized as follows. In Section II, we present opportunistic scheduling scheme that is proposed in [1] in more detail. In Section III, we present our opportunistic scheduling and adaptive modulation. We provide numerical results in Section IV and finally conclude in Section V. 

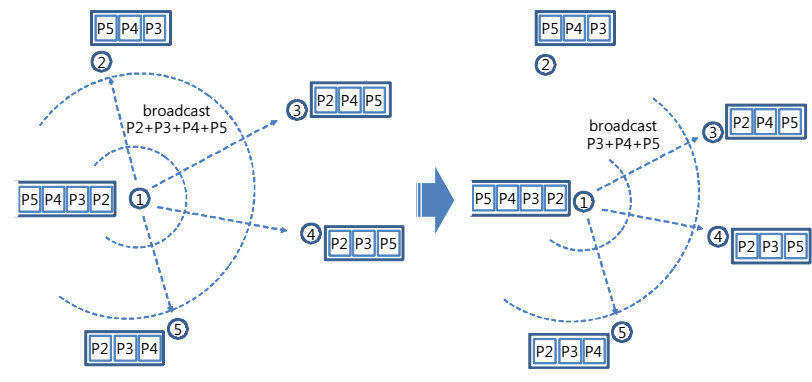

Fig. 3. The opportunistic scheduling of the network coding group

\section{OPPORTUNISTIC SCHEDULING WITH AN IDEALIZED SYSTEM}

In [1], the capacity of each link to destination node $i$ is modeled with Shannon capacity as

$$
C_{i}=\log _{2}\left(1+\gamma_{i}\right)
$$

where $\gamma_{i}$ is the instantaneous signal to noise ratio (SNR) at destination node $i$. Hence, it is assumed that if the data rate at which a destination node receives is below its capacity, then data can be transmitted without any error and otherwise, data cannot be transmitted at all. Since a coded packet is transmitted to multiple destination nodes, to be transmitted to all destination nodes without errors, its transmission data rate should be determined by the SNR of the destination node with the lowest SNR. Hence, assuming that destination nodes in the network coding group are ordered in an increasing order of their SNR's, the instantaneous throughput for the coded packet that includes native packets for destination nodes whose SNR are higher than or equal to the $k$ th lowest SNR in the network coding group is defined as

$$
C_{\text {inst }}^{k}=(N-k+1) \log _{2}\left(1+\gamma_{k}\right), k=1,2, \cdots, N,
$$

where $N$ is the number of destination nodes in the network coding group. Hence, to maximize the instantaneous throughput for the transmission of the coded packet, a node chooses the optimal scheduling level $k_{\text {opt }}$ as:

$$
k_{\text {opt }}=\arg \max _{k} C_{i n s t}^{k} .
$$

Once the optimal scheduling level $k_{\text {opt }}$ is determined, the node makes a coded packet including only native packets for destination nodes whose SNR are higher than or equal to $k_{\text {opt }}$ th lowest SNR in the network coding group. Hence, native packets are scheduled for network coding considering the channel condition at each destination node in the network coding group. Since the optimal number of scheduled native packets is dynamically changed depending on time-varying SNR's at destination nodes in the network coding group, the MAC-optimal coding option that includes all destination nodes in the network coding group might not provide the maximum throughput.

For example, in Fig. 3, node 1 wants to transmit packets $P 2, P 3, P 4$, and $P 5$ to nodes 2, 3, 4, and 5, respectively. In this example, the MAC optimal coding option is to include all packets, i.e., selecting all destination nodes, in a coded packet. However, if the SNR at node 2 is too low, the data rate for a reliable transmission for the coded packet might be too low, since it depends on the low SNR at node 2. Hence, in this case, it might be a better choice that maximize the throughput to exclude $P 2$ to node 2 from the coded packet and transmit $P 3+P 4+P 5$ with a higher data rate only to nodes 3,4 , and 5 .

\section{PROPOSED OPPORTUNISTIC SCHEDULING AND ADAPTIVE MODULATION}

In this section, we study an opportunistic scheduling and adaptive modulation problem given the network coding group that consists of a transmitter node and destination nodes that satisfy conditions for the network coding group in Section I. We assume that there are $N$ destination nodes (e.g., nodes 2, 3, 4, and 5 in Fig. 3) in the network coding group and they are ordered in a decreasing order of their SNR's. Hence, destination node 1 has the highest SNR while destination node $N$ has the lowest SNR. We assume that the transmission power of a transmitter node (e.g., node 1 in Fig. 3 ) is fixed to be $P_{t x}$. Then, the SNR at each destination node $i$ at time $t$ is obtained as

$$
\gamma_{i}[t]=P_{t x}\left(\frac{h_{i}[t]}{\sigma_{i}[t]}\right)^{2},
$$

where $h_{i}[t]$ and $\sigma_{i}[t]$ are complex channel gain and standard deviation of AWGN at the destination node $i$ at time $t$, respectively. Since we focus on the throughput of a single transmission that occurs at a specific time $t$, we omit the index for time, $t$, in the following. If we assume that each link $i$ experiences Rayleigh fading, the probability density function of the SNR at each destination node $i$ is given as [7]:

$$
p\left(\gamma_{i}\right)=\frac{1}{\gamma_{a v, i}} e^{-\gamma_{i} / \gamma_{a v, i}},
$$

where $\gamma_{a v, i}$ is the average SNR at destination node $i$. We assume that the transmitter node knows the instantaneous SNR at each destination node.

We define the expected throughput at destination node $i$ given constellation size of modulation $M_{i}$ and SNR $\gamma_{i}$, $r_{i}\left(M_{i}, \gamma_{i}\right)$, as the expected number of bits that can be transmitted without errors in a symbol duration. Hence, it is obtained as

$$
r_{i}\left(M_{i}, \gamma_{i}\right)=\left(\log _{2} M_{i}\right)\left(1-p_{e}\left(M_{i}, \gamma_{i}\right)\right),
$$

where $p_{e}\left(M_{i}, \gamma_{i}\right)$ is the error probability at node $i$, which is a function of $M_{i}$ and $\gamma_{i}$. For example, with M-PSK, the error probability is given as [7]

$$
p_{e}\left(M_{i}, \gamma_{i}\right)=2 Q\left(\sqrt{2 \gamma_{i}} \cdot \sin \frac{\pi}{M_{i}}\right),
$$

where $Q(x)=\frac{1}{\sqrt{2 \pi}} \int_{x}^{\infty} e^{-\frac{t^{2}}{2}} d t$. With M-QAM, the error probability is given as [7]

$$
p_{e}\left(M_{i}, \gamma_{i}\right)=4 A\left(M_{i}, \gamma_{i}\right) \cdot\left(1-A\left(M_{i}, \gamma_{i}\right)\right),
$$


where $A\left(M_{i}, \gamma_{i}\right)=\left(1-\frac{1}{\sqrt{M_{i}}}\right) \cdot Q\left(\sqrt{\frac{3 \gamma_{i}}{M-1}}\right)$.

We now define the expected throughput for the transmission of a coded packet given that it includes native packets for destination nodes $1,2, \cdots, n$ and $\bar{\gamma}^{n}=\left(\gamma_{1}, \gamma_{2}, \cdots, \gamma_{n}\right)$ as the sum of the expected throughput at destination node $i$ given $\gamma_{i}$ that are included in the coded packet. Hence, if a coded packet includes native packets for destination nodes $1,2, \cdots, n$, it is obtained as

$$
r^{n}\left(M, \bar{\gamma}_{n}\right)=\left(\log _{2} M\right) \sum_{i=1}^{n}\left(1-p_{e}\left(M, \gamma_{i}\right)\right),
$$

where $M$ is the constellation size of modulation of the coded packet. In this paper, we will maximize the expected throughput for the transmission of a coded packet given SNR vector $\bar{\gamma}$ of destination nodes in the network coding group by optimally choosing destination nodes, i.e., $n$ and constellation size of modulation, i.e., $M$ of the coded packet in (9).

We first study how to obtain the optimal selection of destination nodes within the coding packet given SNR vector $\bar{\gamma}$, i.e., the optimal scheduling policy.

Proposition 1: For any given $\bar{\gamma}=\left(\gamma_{1}, \gamma_{2}, \cdots, \gamma_{N}\right)$, the optimal scheduling policy for maximizing the expected throughput for the transmission of a coded packet is to include native packets for all destination nodes in the network coding group.

Proof: First, note that the expected instantaneous throughput at destination node $i, r_{i}\left(M_{i}, \gamma_{i}\right)$, is nonnegative for any $M_{i}$ and $\gamma_{i}$. Let us define $M_{o p t}^{n}\left(\bar{\gamma}_{n}\right)$ as the optimal constellation size when the coded packet includes native packets only for destination nodes $1,2, \cdots, n$. Then, the expected throughput for the transmission of the coded packet defined in (9) is obtained as

$$
\begin{aligned}
& r^{n}\left(M_{o p t}^{n}\left(\bar{\gamma}_{n}\right), \bar{\gamma}_{n}\right) \\
= & \left(\log _{2} M_{o p t}^{n}\left(\bar{\gamma}_{n}\right)\right) \sum_{i=1}^{n}\left(1-p_{e}\left(M_{o p t}^{n}\left(\bar{\gamma}_{n}\right), \gamma_{i}\right)\right) .
\end{aligned}
$$

Hence,

$$
\begin{aligned}
& r^{n}\left(M_{o p t}^{n}\left(\bar{\gamma}_{n}\right), \bar{\gamma}_{n}\right) \\
\leq \quad & \left(\log _{2} M_{o p t}^{n}\left(\bar{\gamma}_{n}\right)\right) \sum_{i=1}^{n}\left(1-p_{e}\left(M_{o p t}^{n}\left(\bar{\gamma}_{n}\right), \gamma_{i}\right)\right) \\
& +\log _{2} M_{o p t}^{n}\left(\bar{\gamma}_{n}\right)\left(1-p_{e}\left(M_{o p t}^{n}\left(\bar{\gamma}_{n}\right), \gamma_{n+1}\right)\right) \\
= & \left(\log _{2} M_{o p t}^{n}\left(\bar{\gamma}_{n}\right)\right) \sum_{i=1}^{n+1}\left(1-p_{e}\left(M_{o p t}^{n}\left(\bar{\gamma}_{n}\right), \gamma_{i}\right)\right) \\
= & r^{n+1}\left(M_{o p t}^{n}\left(\bar{\gamma}_{n}\right), \bar{\gamma}_{n+1}\right) \\
\leq & r^{n+1}\left(M_{o p t}^{n+1}\left(\bar{\gamma}_{n+1}\right), \bar{\gamma}_{n+1}\right) .
\end{aligned}
$$

Hence, given $\bar{\gamma}, r^{n}\left(M_{o p t}^{n}\left(\bar{\gamma}_{n}\right), \bar{\gamma}_{n}\right)$ is nondecreasing in $n$. This implies that the optimal scheduling policy is to include native packets for all destination nodes in the network coding group in the coded packet.

Since for any given $\bar{\gamma}$, the optimal scheduling is selecting all destination nodes in the network coding group, we can obtain the optimal constellation size for given $\bar{\gamma}$ by solving

$$
M_{\text {opt }}(\bar{\gamma})=\arg \max _{M} r^{N}\left(M, \bar{\gamma}_{N}\right) .
$$

By substituting the error probability of the modulation scheme that is used in the system, e.g., (7) for M-PSK (8) for MQAM, into (9), the expected throughput for the transmission of the coded packet for each constellation size can be calculated. Since the number of possible constellation sizes for a given modulation scheme is not too large, the above problem can be solved easily.

Since the SNR at each destination node is a random variable, we can calculate the expected instantaneous throughput for the transmission of a coded packet by using the joint pdf of SNR's as

$$
r_{a v}=\int \ldots \int r_{N}^{N}\left(M_{o p t}(\bar{\gamma}), \bar{\gamma}\right) p(\bar{\gamma}) d \gamma_{1} \cdots d \gamma_{N},
$$

where $p(\bar{\gamma})$ is the joint pdf of SNR's at destination nodes in the network coding group. When all SNRs at destination nodes are independent of each other and Rayleigh distributed, the joint pdf of SNR's is obtained as a product of each marginal pdf in (5):

$$
p(\bar{\gamma})=\prod_{i=1}^{N}\left(\frac{1}{\gamma_{a v, i}} e^{-\gamma_{i} / \gamma_{a v, i}}\right) .
$$

\section{NumericAl RESUlts}

In this section, we present simulation results for our scheduling and adaptive modulation scheme. Throughout this section, we model wireless links with i.i.d. Rayleigh distributions.

In Figs. 4 and 5, we first validate the optimality of our scheduling policy in Proposition 1 for M-PSK and MQAM, respectively. In these figures, we consider a network coding group with the number of destination nodes, $N=10$. We vary the average SNR at destination nodes and plot the expected throughput for the transmission of a coded packet for each different number $k$ of destination nodes in the coded packet When the coded packet has $k$ destination nodes, $k$ destination nodes with higher SNR's among $N$ destination nodes in the network coding group are selected. In addition, for given $k$ destination nodes, the constellation size of each modulation scheme is optimally selected. As shown in these figures, as $k$ increases, the expected throughput of the coded packet also increases, which validates the optimality of our scheduling policy.

We now compare the performance of our adaptive modulation scheme with the scheme with fixed modulation, in which its constellation size is fixed regardless of SNR's at destination nodes. In Figs. 6 and 7, we consider M-PSK and MQAM, respectively, with the network coding group with $N=5$. We assume that possible constellation sizes are $4,8,16,32$, and 64 . As shown in these figures, our scheme always provides a higher expected throughput than the scheme with a fixed constellation size. As the average SNR increases, the best constellation size for the scheme 


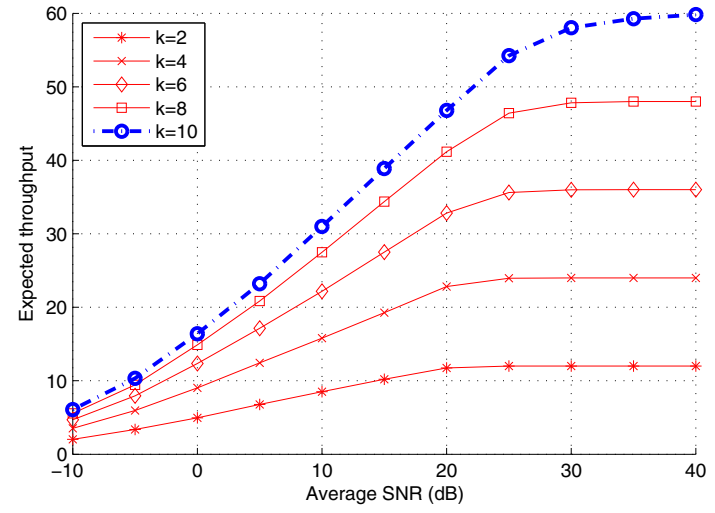

Fig. 4. The expected throughput for the transmission of a coded packet with a different number of destination nodes in the coded packet for M-PSK.

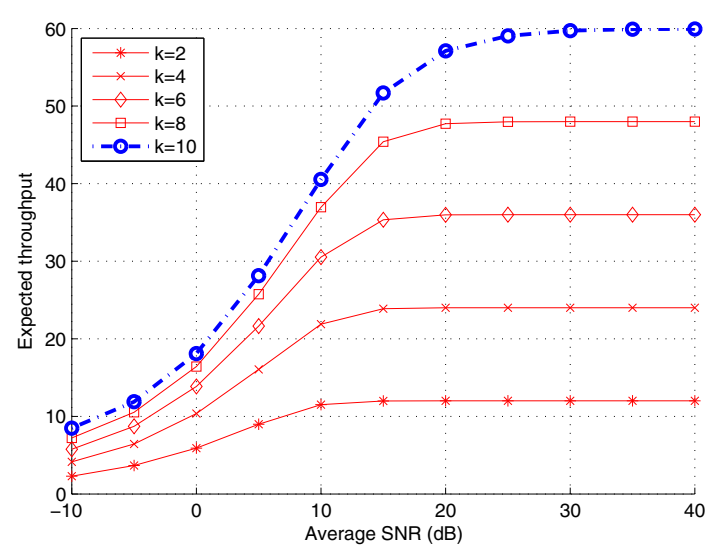

Fig. 5. The expected throughput for the transmission of a coded packet with a different number of destination nodes in the coded packet for MQAM.

with a fixed constellation size also increases. This implies that to maximize the throughput for the transmission of a coded packet, it is important to consider the channel condition appropriately at each destination within the coded packet.

\section{CONCLUSION}

In this paper, we studied an opportunistic scheduling and adaptive modulation problem for wireless networks with network coding, in which native packets that are encoded in a coded packet and the modulation level of a coded packet are selected based on the channel condition at the destination node for each native packet. We showed that to maximize the throughput of a transmission, all available native packets should be selected regardless of channel conditions of destination nodes for native packets, while the constellation size for modulation should be adjusted based on channel conditions of destination nodes for native packets.

\section{REFERENCES}

[1] H. Yomo and P. Popovski, "Opportunistic scheduling for wireless network coding," IEEE International Conference on Communications, pp. 5610$5615,2007$.

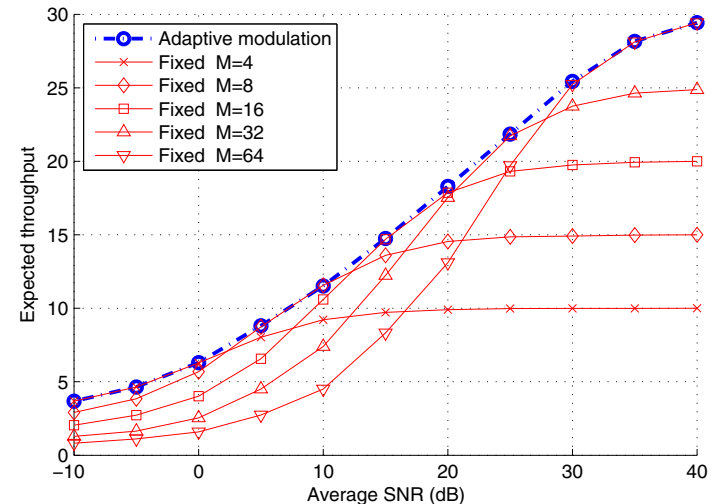

Fig. 6. Comparison of adaptive modulation and fixed modulation for M-PSK.

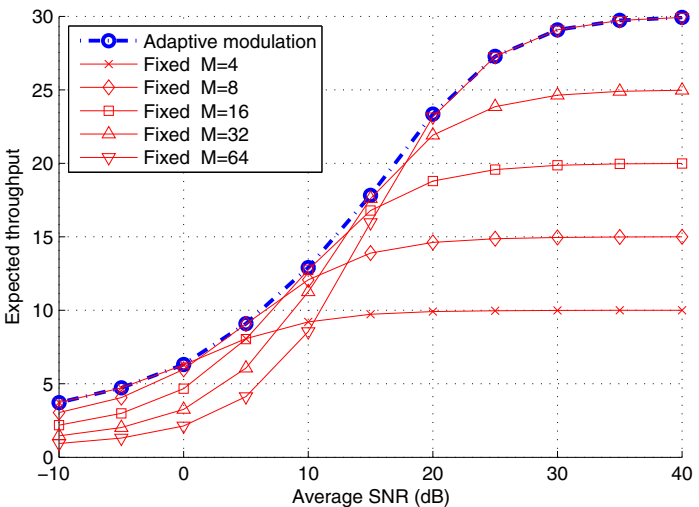

Fig. 7. Comparison of adaptive modulation and fixed modulation for MQAM.

[2] R.Ahlswede, N.Cai, S.-Y. R. Li, and R. W. Yeung, "Network information flow," IEEE Transactions on Information Theory, vol. 46, no. 4, pp. 12041216, 2000.

[3] Y. Wu, Network coding for wireless networks. Microsoft Research Technical Report MSR-TR-2007-90, 2007.

[4] C. Fragouli, D. Katabi, A. Markopoulou, M. Medard, and H. Rahul, "Wireless network coding: Opportunities and challenges," Military Communications Conference, pp. 1-8, 2007.

[5] S. Katti, H. Rahul, W. Hu, D. Katabi, M. Medard, and J. Crowcroft, "Xors in the air: Practical wireless network coding," IEEE/ACM Transactions on Networking, vol. 16, no. 3, pp. 497-510, 2008.

[6] S. Katti, D. Katabi, W. Hu, R. Hariharan, and M. Medard, "The importance of being opportunistic: Practical network coding for wireless environments," Proc. 43rd Allerton Conf. on Communication, Control, and Computing, 2005.

[7] J. G. proakis and M. Salehi, Digital Communications, 4th ed. McGrawHill, 2000. 